\title{
Sonderleistung und Sondervergütung
}

\section{ArbG Essen, Urteil vom 9. Dezember 1982 (3 Ca 1932/82)}

\section{Nichtamtlicher Leitsatz}

Zu den Voraussetzungen für eine zusätzliche Vergütung für eine Sonderleistung.

\section{Paragraphen}

BGB. \& 242, \$612

\section{Stichworte}

Sonderleistung/-vergütung

\section{Tatbestand}

Der Kläger war bei der Beklagten vom 1. Oktober 1968 bis 31. Dezember 1980 (unter anderem) als Projektmanager“ für den Bau von Hüttenwerken „zu einem Monatsgehalt von zuletzt 5600,- DM brutto tätig.

Mit seiner Klage fordert er von der Beklagten für die Nutzung des von ihm entwickelten Informationssystems IBOS eine (Sonder-) Vergütung in Höhe von $100000,-$ DM.

Der Kläger trägt hierzu vor: Mit der Entwicklung des Informationssystems IBOS habe er eine Leistung schöpferischer Art erbracht. Er habe ein handliches und effizientes Informationssystem geschaffen, mit dem der gewaltige bei der Planung und Realisierung großer Anlagenprojekte freigesetzte Informationsfluß ohne Schwierigkeiten in Griff zu halten sei.

Bei der Entwicklung des Informationssystems IBOS habe es sich um eine echte Sonderleistung gehandelt, die über die von ihm gegenüber der Beklagten geschuldete Arbeitsleistung hinausgegangen sei.

Nachdem er die Beklagte mehrfach auf Mängel der beim Lieferauftrag Hüttenwerk Spanien verwandten Nomenklatur aufmerksam gemacht habe, habe er der Beklagten mit Schreiben vom 13. Juli 1970 das von ihm privat entwickelte Informationssystem FAN (Funktionelle-Anlagen-Nomenklatur) angeboten.

Mit Schreiben vom 7. August 1970 habe die Beklagte die ,Zuständigkeit zur Erarbeitung von Nummern- und Schlüsselsystemen für die Projekt- und Auftragsabwicklung Industrieanlagen und Herausgabe entsprechender Arbeitsrichtlinien" der Zentralabteilung Technische Koordination zugewiesen.

Nach Erhalt des Schreibens habe der Kläger nur noch privat an einer unternehmens- und anlagenneutralen Lösung des Problems der Informationssteuerung, -bündelung und -darbietung gearbeitet. In den folgenden Jahren habe er schließlich ein Projekt-Management-System für die Führung, Koordination, Planung und Realisierung von Industrieanlagen-Projekten jeder Art und Größe entwickelt (das ,InformationsBündel-Organisations-System' mit der Kurzbezeichnung , $\mathrm{IBOS}^{\prime}$ ).
Der Kläger habe allerdings zugelassen, daß sein Informationssystem IBOS - erstmals 1972 - von der Zentralabteilung Technische Koordination und von einzelenen Projektleitern versuchsweise angewandt wurde.

Im April 1974 habe der Kläger die von der Beklagten gestellte Frage bejaht, ob sein Nummern- und Informationssystem auch für einen so großen Auftrag wie den Bau eines integrierten Hüttenwerkes in Mittelrußland anwendbar sei.

Daraufhin habe ihn die Baklagte in der Zeit vom 1. Juni 1974 bis 30. September 1976 als ,Koordinator für Informatik und Leiter der Informationsstelle ${ }^{6} \mathrm{zu}$ der aus drei deutschen Firmen gebildeten Arbeitsgemeinschaft entsandt, die von den sowjetischen Ministerien zunächst mit der Erstellung eines Vorprojektes beauftragt worden war.

Als einen ihrer Beiträge habe die Beklagte das vom Kläger entwickelte Systeun IBOS in die Vorplannng eingebracht.

Im Jahre 1977 habe der Kläger erstmals bei der Beklagten mündlich wegen einer Vergütung für die Nutzung seines Informationssystems vorgesprochen.

Mit Schreiben vom 9. April 1979 habe die Beklagte festgelegt, daß für diesen Auftrag die FAC-Codierung und das IBOS-Informationssystem anzuwenden seien (dies gelte im übrigen auch für den ProjektleitungsLieferauftrag bezüglich des integrierten Hüttenwerkes in Libyen).

Mit ,Rundschreiben Nr. 32/79“ habe die Beklagte mitgeteilt, daß ab 1. Januar 1980 eine Zentralabteilung Projektbegleitung eingerichtet werde, zu deren Aufgabe u.a. gehöre

- Weiterentwicklung und Anwendung des Informations-Bündelungs- und Organisationssystems (IBOS) und anderer Systeme.

Durch den Einsatz des Informationssystems IBOS habe die Beklagte einen nicht unerheblichen Vorteil erlangt, da hierdurch das Planungs- und Realisierungsrisiko gesenkt werde.

Der wirtschaftliche Wert von IBOS werde u.a. dadurch bewiesen, daß die Beklagte mit Schreiben vom 16. Mai 1978 an die Firma GmbH die ,Benutzung des IBOS‘ für $228000,-$ DM angeboten habe.

Die Beklagte beruft sich (u.a.) auf die Gesichtspunkte der Verjährung und Verwirkung.

Außerdem weist sie darauf hin, daß der Kläger am Jahresende jeweils, Sonderzahlungen' erhalten habe (Beträge zwischen 6600,- und 9900,- DM)."

Entscheidungsgründe:

„Die Klage ist begründet. Der Kläger hat gegen die Beklagte einen Anspruch auf Zahlung von 100000,DM aus den $\$ \S 242,611,612$ BGB. 
I. Da die Sonderregelungen des Arbeitnehmererfindungsgesetzes, des Urhebergesetzes und des firmeninternen Vorschlagswesens hier nicht eingreifen, kann auf die generelle Norm des $\S 242$ BGB (Grundsatz von Treu und Glauben) zurückgegriffen werden.

II. Hiernach hat der Kläger einen Anspruch auf eine zusätzliche Vergütung unter folgenden Voraussetzungen (vergl. Urteil des Bundesarbeitsgerichtes vom 30. April 1965, GRUR 1966, Seite 88):

1) Der Arbeitnehmer muß eine Leistung schöpferischer Art erbracht haben.

2) Es muß sich um eine (Sonder-) Leistung gehandelt haben, die nicht im Aufgabenbereich des Arbeitnehmers gelegen hat und deshalb nicht als durch das Gehalt mitabgeholten anzusehen ist.

3) Der Arbeitgeber muß diese Leistung genutzt und dadurch einen nicht unerheblichen Vorteil erlangt haben.

Die genannten Voraussetzungen sind hier erfüllt:

zu 1) Der Kläger hat mit der Entwicklung des Informationssystems IBOS (und der dazu gehörigen FACListen) eine Leistung schöpferischer Art erbracht.

Er hat ein in sich geschlossenes System entwickelt, mit dem die bei der Planung und Realisierung großer Anlagenprojekte anfallende Informationsmenge bewältigt werden kann.

Die Beklagte hat selbst zu erkennen gegeben, daß auch sie das Informationssystem IBOS für ein schöpferisches Werk (von eigenem wirtschaftlichen Wert) hält:

Sie hat nämlich mit Schreiben vom 16. Mai 1978 die ,Benutzung des IBOS ${ }^{\star}$ an ein anderes Unternehmen zum Preise von $228000,-$ DM angeboten.

zu 2) Die Entwicklung des Informatiunssystems IBOS gehörte nicht zu den vom Kläger arbeitsvertraglich geschuldeten Tätigkeiten.

Die Beklagte hat den Kläger im Jahre 1968 als ,Projektmanager eingestellt, ohne dessen Arbeitsaufgabe näher zu beschreiben.

$\mathrm{Zu}$ den Aufgaben eines Projekmanagers gehört es sicherlich, auf bestebende Informationssysteme zurückzugreifen und diese - unter Anpassung an die besonderen betrieblichen Verhältnisse - anzuwenden.

Etwas anderes gilt indessen für die Entwicklung (in sich geschlossener) neuer Informationssysteme. Insoweit hätte es einer besonderen arbeitsvertraglichen Verpflichtung des Klägers bedurft.

Der Kläger hat in diesem Zusammenhang umfangreiches Tatsachenmaterial zusammengetragen. Die Richtigkeit seiner Beurteilung ergibt sich jedoch bereits aus dem eigenen Verhalten der Beklagten:

So hat die Beklagte mit Rundschreiben vom 7. August 1970 die ,Zuständigkeit zur Erarbeitung von Nummern- und Schlüsselsystemen für die Projektund Auftragsabwicklung Industrieanlagen " nicht den einzelnen Projektleitern, sondern der Zentralabteilung ,IT-Technische Koordination' zugewiesen.

Weiterhin hat die Personalabteilung der Beklagten mit Schreiben vom 26. Juni 1980 an die Abteilung Vorschlagswesen mitgeteilt, daß die Frage, ob die Entwicklung eines Informationssystems unter das Aufgabengebiet des Klägers falle, aus der Personalakte nicht beantwortet werden können. zu 3) Die Beklagte hat das vom Kläger entwickelte Informationssystem IBOS (u. a.) bei der Durchführung des (Rußland) Vorprojektes eingesetzt. Weiterhin findet das System Anwendung bei der Abwicklung des Lieferauftrages.

Der nicht unerhebliche wirtschaftliche Vorteil der Beklagten liegt bereits darin, daß die Beklagte gegenüber dem Auftraggeber zur Verwendung des Informationssystems vertraglich verpflichtet ist und mit der Nutzung des Systems eben dieser vertraglichen Verpflichtung nachkommt.

II. Hinsichtlich der Hèhe der Vergütung erscheinen dem Gericht die vom Kläger geforderten $100000,-$ DM als angemessen (vergl. § 612 Abs. 2 BGB).

Hier ist erneut auf die Tatsache hinzuweisen, daß die Beklagte im Jahre 1978 die ,Benutzung des IBOS einem anderen Unternehmen zum Preis von 228 000,$\mathrm{DM}$ angeboten hat.

Die vom Kläger entwickelte Größenvorstellung bewegt sich somit eher an der untensten Grenze.

III. Die Beklagte hat den Anspruch des Klägers auf Zahlung einer Sondervergütung für die Nutzung des Informationssystems IBOS nicht durch die von ihr erbrachten jährlichen Sonderzahlungen erfüllt.

Diese Sonderleistungen beruhten auf den arbeitsvertraghichen Vereinbarungen der Parteien. Hier geht es indessen um die Vergütung für eine über die arbeitsvertraglichen Verpflichtungen des Klägers hinausgehende Sonderleistung.

IV. Der Anspruch des Klägers ist weder verwirkt noch verjährt.

Diese Einwendungen und Einreden der Beklagten scheitern schon daran, daß die Beklagte das vom Kläger entwickelte Informationssystem IBOS auch heute noch nutzt.

Da die Nutzung der vom Kläger erbrachten Sonderleistung - dies ist eine Tatbestandsvorassetzung für das Bestehen des Anspruchs des Klägers - somit in der Gegenwart liegt, ist die Fälligkeit des hier streitigen Anspruchs (auch) in der Gegenwart, nicht aber (ausschließlich) an längst vergangenen Zeitpunkten eingetreten.“

\section{Anmerkung}

(1) Die Parteien haben sich vor dem LAG auf einen Betrag von $60000,-$ DM verglichen.

(2) Das Urteil wird trotz seines Alters veröffentlicht, weil Buchner es zum Ausgangspunkt seines Aufsatzes „Die Vergütung für Sonderleistungen des Arbeitnehmers - ein Problem der Äquivalenz der im Arbeitsverhältnis $\mathrm{zu}$ erbringenden Leistungen “ in GRUR 1985, 1 ff. (mit falscher Angabe des Geschäftszeichens) gemacht hat, aber m.E. den Sachverhalt nieht richtig wiedergegeben hat. Auf sachlich falscher Grundlage kritisiert er, daß es sich nicht um eine Sonderleistung handeln würde. Er übersieht, daß der Kläger unwidersprochen vorgetragen hat, daß er das System im wesentlichen privat entwickelt hat. Insofern liegt eine eingebrachte Leisteng vor, die als Sonderleistung anzuerkennen ist.

(ch. z.) 